\title{
Diagnostic criteria should be considered when reviewing the effect of diabetes prevention studies
}

\author{
Hao $\mathrm{Li}^{1} \cdot$ Shen Tian ${ }^{1} \cdot$ Juan $\mathrm{Wu}^{1} \cdot \mathrm{Zhou} \mathrm{Xu}^{1} \cdot$ Ling-quan Kong $^{1}$ \\ Received: 3 August 2019 / Accepted: 6 August 2019 / Published online: 13 September 2019 \\ (C) Springer-Verlag GmbH Germany, part of Springer Nature 2019
}

Keywords Cardiovascular complications - Diabetes prevention study · Fasting plasma glucose $\cdot$ Impaired glucose tolerance Vascular complications

\author{
Abbreviations \\ FPG Fasting plasma glucose \\ IFG Impaired fasting glucose \\ IGT Impaired glucose tolerance
}

To the Editor: Nathan and colleagues recently published a review of diabetes prevention trials, specifically looking at their long-term effects on reducing the diabetes-related vascular complications [1]. Data from nine diabetes prevention studies were thoroughly and comprehensively analysed to investigate the association of diabetes prevention with microvascular or cardiovascular complications. The majority of participants enrolled in the nine studies had either impaired glucose tolerance (IGT) or impaired fasting glucose (IFG) or both. We found that in Table 1 of their review [1], Nathan et al included details of the specific diagnostic criterion for IFG, which was a fasting plasma glucose (FPG) level of 5.3-6.9 $\mathrm{mmol} / 1$ in the Diabetes Prevention Program (DPP) trial, but no details were given for other trials. In fact, the definitions given for IFG or IGT in terms of the range of FPG were inconsistent across studies.

We have adjusted the table to provide the FPG ranges of the definitions (Table 1). The definition of IGT in terms of $2 \mathrm{~h}$ plasma glucose $(7.8-11.0 \mathrm{mmol} / \mathrm{l})$ was the same in nine studies, but the diagnostic criterion for IFG or IGT in term of FPG was different across studies. In particular, in the Da Qing Diabetes Prevention Study (DQDPS), Finnish Diabetes Prevention Study (FDPS) and the Study to Prevent NonInsulin-Dependent Diabetes Mellitus (STOP-NIDDM), some of the original participants who were enrolled decades ago with an FPG of 7.0-7.7 mmol/1 should have been diagnosed with diabetes, rather than prediabetes, according to the current diabetes diagnostic criterion as set out in 1999 [2]. Reviewing these diabetes prevention cohorts without a concrete interpretation of the diagnostic criteria may, to some extent, lead to an insufficient understanding of their original results. These minor differences in the IFG or IGT diagnostic criterion according to FPG may at least partly explain the discrepant results between these diabetes prevention studies. Therefore, we have added the information regarding the criteria in Table 1 as a supplement to the work by Nathan et al.

Ling-quan Kong

huihuikp@163.com

1 Department of Endocrine and Breast Surgery, The First Affiliated Hospital of Chongqing Medical University, Chongqing 400016, China 


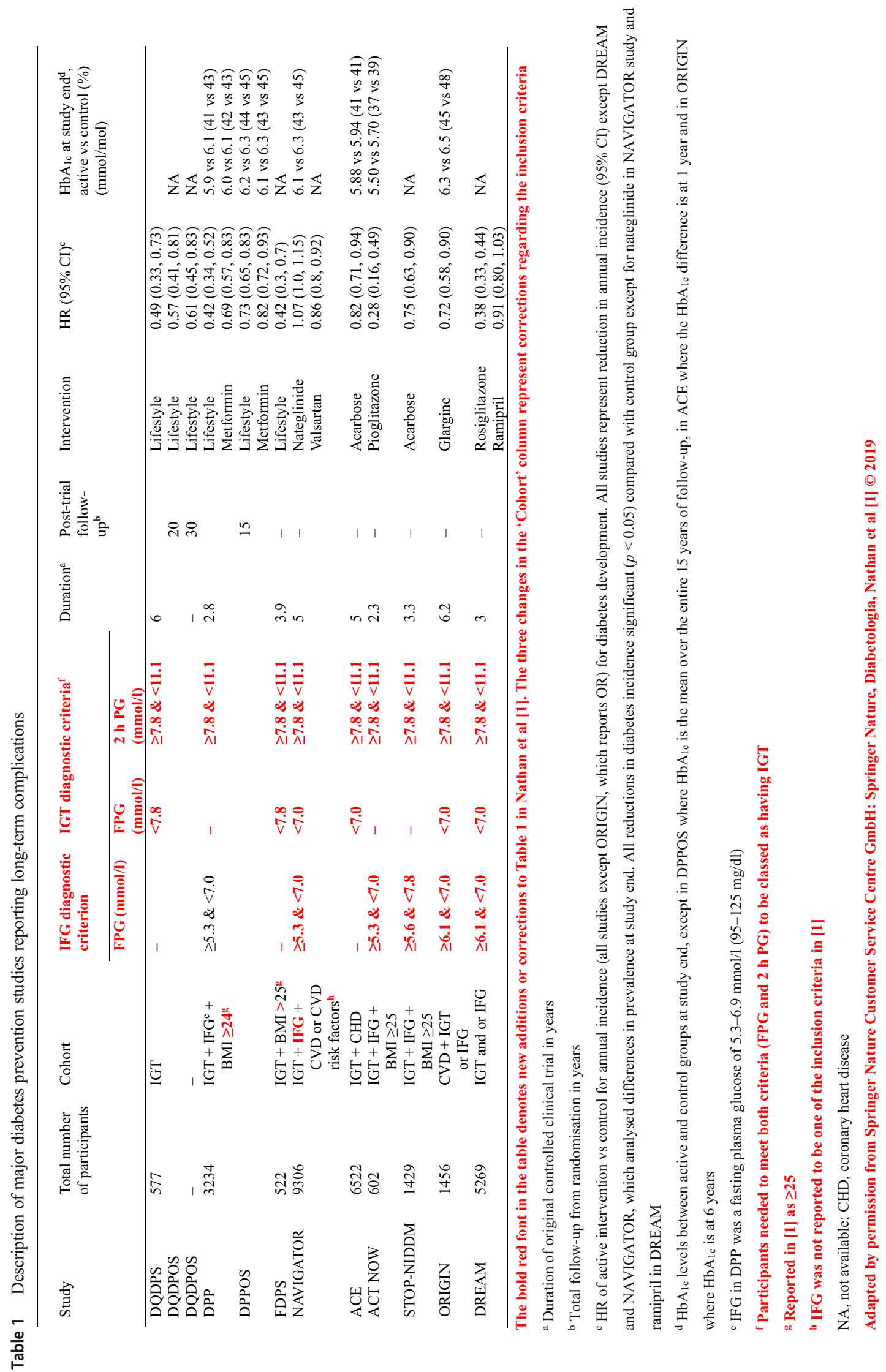


Acknowledgements We sincerely thank Y.-h. Pu for her helping in language improvement and moral support.

Duality of interest The authors declare that there is no duality of interest associated with this manuscript.

Contribution statement All authors were responsible for drafting the article and revising it critically for important intellectual content. All authors approved the version to be published.

\section{References}

1. Nathan DM, Bennett PH, Crandall JP et al (2019) Does diabetes prevention translate into reduced long-term vascular complications of diabetes? Diabetologia 62(8):1319-1328. https://doi.org/10.1007/ s00125-019-4928-8

2. WHO, International Diabetes Federation (2006) Definition and diagnosis of diabetes mellitus and intermediate hyperglycemia: report of a WHO/IDF consultation. World Health Organization, Geneva

Publisher's note Springer Nature remains neutral with regard to jurisdictional claims in published maps and institutional affiliations. 